\title{
A new formula for the rotation velocity - and density distribution of a galaxy
}

\author{
Tony Barrera, Bo Thelin \\ Barrera Kristiansen AB Granitvägen 12B, S-752 43 Uppsala, Sweden \\ Solarphotonics HB Granitvägen 12B, S-752 43 Uppsala, Sweden
}

\begin{abstract}
In this paper a new formula about the formation of galaxies is presented. It is based on the relativistic Schwarzschild metric, where it has been possible to obtain expressions for the rotation velocity - and mass distribution versus distance to the galactic center.

According to the rotation velocity formula, the rotation velocity raises very rapidly from the center and reach a plateau which is constant out to big distance from the center. This is in accordance with observations and is also in accordance with the main structure of rotation velocity versus distance relationships from different galaxy measurements.

The density distribution formula shows that the density of the galaxy will decrease versus distance which is in accordance to observations.

Computer simulations were also performed to establish and verify the rotation velocity and density distributions in the galaxy, according to this paper.
\end{abstract}

Keywords: Astrophysics, Spiral galaxies and Theory of Relativity

\section{Introduction}

The mass of a spiral galaxy can be determined from the dependence of its rotational velocity as a function of the distance from the center of the galaxy. Such a rotational curve has been determined from gas and stars in the distant parts of our galaxy, far beyond our distance to the center. Unexpectedly, it does not follow the Keplerian decrease in which the circular rotation velocity $\mathrm{v}$ decreases $\alpha \mathrm{R}^{-1 / 2}$ where $\mathrm{R}$ is the distance to the center. According to the 3:rd law of Kepler the mass of a galaxy can be expressed as : $\quad M=v^{2} R / G$ and the rotation velocity as : $v=(G M / R)^{1 / 2}$ where $G$ is the gravitation constant.

By using these formulas it is possible to determine the mass and rotation speed at a certain distance of the galaxy. According to these formulas both mass and rotation velocity will decrease with increasing distance.

In the 1970s and 1980s radio astronomers discovered that the spiral rotation velocity remains constant with increasing radius Freeman (1) ,Rubin and Ford (2). They studied neutral hydrogen clouds at 21-cm radio wavelength and in the optical wavelength in spiral galaxies and found non-Keplerian rotation curves.

This is illustrated in Combes et al.(3) (Figs 3.1-3.3) , where the velocities of many spiral galaxies increase the velocity very rapidly at small distances up to a constant plateau at larger distances from the galaxy center. Astronomers discovered that many galaxies rotated at very high velocities.

To explain this most astronomers believe that this is caused by introducing dark matter in the Keplerian equations above and to keep the galaxies together. They believe that most matter in a galaxy consists of dark matter and only a minor part consists of ordinary matter which emits light. As constant rotation velocity is observed now, means that the mass (visible + invisible) increase with distance from the center according to Keplers equations above. Many astronomers believe that much invisible dark matter is situated outside the visible part of the galaxy Ostriker et al. (4) and Einasto et al. (5). They found that M increased linearly with R out to at least $100 \mathrm{kpc}$. They now believe that around $90 \%$ of the mass in a spiral galaxy consist of dark and invisible matter, Rubin (6), with a mass to light (luminosity) ratio in solar units : $\mathrm{M} / \mathrm{L} \approx 200\left(\mathrm{M}_{\text {sun }} / \mathrm{L}_{\text {sun }}\right)$.

There is also another possible mass distribution according to the so called King's model Combes et al. (3) (p.87) where spiral galaxies follow a $\mathrm{M} \alpha \mathrm{R}^{2}$ distribution between mass and distance to center, which is observed. This means that there are different ideas about mass distribution which observations seems to follow in some way. The common for these ideas is the lack of ideas how the mass distribution together with constant rotation velocity distribution coincide with observations at big distance observation.

A trial to solve these problems is done by MOND project, Sanders (7), which is a theoretical model which might explain flat curves by modification of Newton's law of gravitation. In this model gravitational attraction of a given mass $\mathrm{M}$ does not decrease as quickly as $1 / \mathrm{R}^{2}$ at these large distances. The acceleration and thus the rotational velocity of gas for a given stellar mass will be greater.

In this very paper we have presented a new formula about the formation of galaxies. It is based on the relativistic Schwarzschild metric, Schwarzschild (8),Einstein (9), where it has been possible to obtain a formula for the rotation velocity and also a density distribution versus distance to the galactic center. Similar rotation 
velocity profiles to our new formula have also been observed from data published in established books in this field. These profiles are in accordance to observations. Computer simulations were also performed to establish and verify the velocity and density distributions suggested in this paper.

According to this rotation velocity formula, the rotation velocity increases very rapidly from the center and reach a plateau which is constant out to big distance from the center. This has also been observed in many papers, Sofue and Rubin(10) and Combes et al. (3).

The density distribution formula shows that the density of the galaxy will decrease versus distance which is in accordance to observations.

As in the MOND project we try to introduce new ways of thinking introducing relativistic metric which seems to explain observations.

\section{Observational data verifying an $\sqrt{ } \mathbf{x}$ - structure of the galaxy rotation velocity formula}

By using the the formulas published the established books Combes et al. (3) and Lang (11) we can state that we could expect to find a formula for the rotation speed versus distance to the center as a $\sqrt{x}$ dependence. Two proofs of that are demonstrated below :

\section{Proof 1}

According to, Lang (11) (p. 55), the ratio between mass and luminosity of a galaxy has the following appearance :

$$
M / L=\text { const } L^{0.35} \text { giving } M=\text { const } L^{1.35}
$$

giving : $\quad \mathbf{L}=$ const $\mathbf{M}^{(\mathbf{1 / 1 . 3 5 )}}=$ const $\mathbf{M}^{\mathbf{0 . 7 4}}$

By using Tully -Fischer`s law, Combes et al. (3), (p. 87 ), between luminosity and the rotation speed of the galaxy gives:

This means that :

$$
\begin{aligned}
& \mathrm{L} \alpha \mathrm{V}^{4} \alpha \mathrm{M}^{0.74} \\
& \mathrm{~V} \alpha \mathrm{M}^{\mathbf{0 . 1 9}}
\end{aligned}
$$

According to Combes et al., (p. 87 ), the correlation between mass and radios is :

$$
\mathbf{M} \alpha \mathbf{R}^{2}
$$

This means that :

$$
\mathrm{V} \alpha \mathrm{M}^{0.19}=\mathbf{R}^{(2 \times 0.19)}=\mathbf{R}^{0.38}
$$

which has $\sqrt{x}$ - structure between rotation velocity and distance to the center of galaxy.

\section{Proof 2}

According to equations (2) and (5) :

$$
\mathbf{L} \alpha \mathbf{M}^{0.74} \alpha \mathbf{R}^{(2 \times 0.74)}
$$

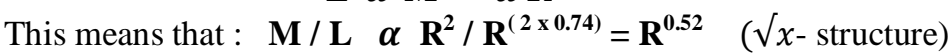

This is observed in Fig( 3.12 ) in Combes et al. (3), (p. 85) and Tinsley (12), where the color index B-V $\alpha$ T $\alpha$ R.

This means that equation (8) can be rewritten as :

means that :

$$
\begin{aligned}
M / L & =\left(V^{(1 / 0.19)} / V^{4)}\right)=V^{1.26}=R^{0.52} \\
V & =R^{0.41}
\end{aligned}
$$

which gives $\sqrt{x}$ - structure. This means that these investigations show that we should expect a rotation velocity profile to have a $\sqrt{x}$ - structure between rotation velocity and distance to the center of the galaxy.

\section{The use of Schwarzschild metric}

\section{A. The determination of the velocity and density formulas}

For a spherically symmetric gravitational field outside a massive non-rotating body in vacuum,the line element ds becomes according to Schwarzschild (8):

$\mathrm{ds}^{2}=\left(1-2 \mathrm{GM} / \mathrm{c}^{2} \mathbf{r}\right) \mathrm{c}^{2} \mathrm{dt}^{2}-\left(1-2 \mathrm{GM} / \mathrm{c}^{2} \mathbf{r}\right)^{-1} \mathrm{dr}^{2}-\mathbf{r}^{2} \mathrm{~d} \theta^{2}-\mathbf{r}^{2} \sin ^{2} \theta$

d $\psi^{2}$

Here $r, \theta$ and $\psi$ are spherical coordinates whose origin is at the center of the massive object with the mass $M$, which determine the Newtonian gravitational field (GM/r). G is here the the gravitational constant and $r$ is the distance. By using a polar coordinate system with $\theta=0$ the following expression from equation (11) is obtained :

$$
d s^{2}=-\gamma^{-1} d r^{2}-r^{2} d \theta^{2}+\gamma d t^{2}
$$

where $\gamma=(\mathbf{1}-\mathbf{2 M} / \mathbf{r})$, which is the Schwarzschild term Eddington (13)( p.82-85) 
If we study galaxies, the expansion in the space-time appeared to be constant, which means that the line element could be $\mathrm{ds}=0$. By using this on equation (12) we obtain :

$$
\mathbf{r}^{2} d \theta^{2}=\gamma d t^{2}-\gamma^{-1} d r^{2}
$$

From this formula it is possible to obtain an expression for the angular rotation speed of a galaxy:

$$
\mathrm{d} \theta=(1 / \mathrm{r})\left((1-2 \mathrm{M} / \mathrm{r}) \mathrm{dt}^{2}-(1-2 \mathrm{M} / \mathrm{r})^{-1} \mathrm{dr}^{2}\right)^{1 / 2}
$$

If we suggest the space-time to be constant for a galaxy the $\mathrm{dr}=0$ which gives

$\mathrm{dr}^{2}=0$. This means that equation (14) can be rewritten as :

$$
\mathrm{d} \theta=(1 / \mathrm{r})\left((1-2 \mathrm{M} / \mathrm{r}) \mathrm{dt}^{2}\right)^{1 / 2}
$$

which gives a simpler equation of the angular rotation speed of galaxies :

$$
\mathrm{d} \theta / \mathrm{dt}=(1 / \mathrm{r})((1-2 \mathrm{M} / \mathrm{r}))^{1 / 2}
$$

By introduction of a scale factor $\mathrm{k}$ equation (16) will get the following

appearance : $\quad \mathbf{d} \boldsymbol{\theta} / \mathbf{d t}=(\mathrm{k} / \mathbf{r})((\mathbf{1}-\mathbf{2 M} / \mathbf{r}))^{\mathbf{1 / 2}}$

The formula of the rotation speed $(\mathrm{km} / \mathrm{s})$ is obtained from the expression

$$
\mathbf{v}=\mathbf{r}(\mathbf{d \theta} / \mathbf{d t})
$$

This means that the rotation speed in $\mathrm{km} / \mathrm{s}$ will be the following expression :

$$
\mathbf{v}=\mathbf{k}((\mathbf{1}-\mathbf{2 M} / \mathbf{r}))^{\mathbf{1} / 2}
$$

and is seen in the theoretical graph of Fig 1 . In this graph the rotation speed is increased steeply at small radius and reach a constant value after a rather small distance. Fig 1 is a theoretical graph only verifying the main structure of equation (19). Similar profiles are also seen in the observational graphs of Fig 2. Many of those graphs follow a clear $\sqrt{x}$-. structure according to chapter A and equation (19) .

\section{B. The determination of the density formula}

By using equation (19) we can now achieve a formula for the mass $M$ and density $\rho$ of the galaxy :

$$
\mathbf{M}=(\mathbf{r} / 2)\left(1-\mathbf{v}^{2} / \mathbf{k}\right)
$$

By using the volume of a sphere :

$$
V=\left(4 \pi r^{3}\right) / 3
$$

the expression of the density of a galaxy is achieved :

$$
\rho=M / V=\left(3 /\left(8 \pi r^{2}\right)\right)\left(1-v^{2} / k\right)
$$

Such a graph is seen in Fig 3 , which is a theoretical graph where the total density of a galaxy is decreasing at larger distancies in accordance to earlier measurements Lang (11) (p.131)

The ratio between $r$ and $R_{\max }$ in percent is here studied.

Computer simulations were also performed to establish and verify the velocity and density distributions suggested in this paper. In these simulations 6000 (Fig 4) and 30000 (Fig 5 and 6) "stars" were randomly distributed like an ellipsoid and following equations (19) and (22). After some time a spiral structure appeared in accordance with observations. Studies were also performed of the halo of the galaxy from the side (Fig 6).

\section{Discussion}

We can se from section 2 that the velocity formula between velocity and distance to the center of the galaxy has a $\sqrt{x}$ - structure. These facts are based on results presented in ( 3 ) and (11) and are not based on Kepler`s 3:rd law directly. These relationships are observed by the astronomers where the velocity reach a constant speed between 5 and $10 \mathrm{kpc}$ from the center of the galaxy. A similar structure is also obtained by using the theory of relativity and the Schwarzschild metric, where a steep raise of the velocity (angular and circular in $\mathrm{km} / \mathrm{s}$ ) at low distancies is obtained by equation (19).After that a plateau is reached which will be dominating to large distancies.

This is also observed by many astronomers (1) and (2) and have been a controversial discovery, because it contradict Kepler`3:rd law, which will follow a $(1 / \sqrt{r})$ - dependence and is not observed in any galaxies. Therefore the astronomers claim that there must be

a big amount of dark matter in the galaxy, which is the cause of this discrepancy and also hold the galaxies together at those high rotation speeds.

Similar $\sqrt{x}$ - structures of the velocity curves as our curves have been obtained in the Mondproject, where a modification of the Newton`s law is applied. This is an interesting approach where an additional term is applied on the usual $1 / \mathrm{R}^{2}$ decrease between gravity and distance on galaxy level. However, we think that applying the theory of relativity here, is a more realistic approach on this galaxy level.

Our model is also in accordance to the density distribution in the galaxy which shows a decreasing profile to distance, which is in accordance to earlier observations. 
Computer simulations were also performed to establish and verify the rotation velocity and density distributions in the galaxy, suggested in this paper and giving a strong support of the new formulas and the use of theory of relativity.

We thank :

\section{Acknowledgement:}

Dr. Gunnar Berg at the Mathematical Institution at Uppsala University,

Prof. Krister Kiselman at the Mathematical Institution at Uppsala University,

Dr. Anders Hast at Creative Media Lab at University of Gävle.

Prof. Ewert Bengtsson at Centre for Image Analysis at Uppsala University

Dr.Staffan Yngve at the Physical Institution at Uppsala University

for valuable discussions and critical examinations of the material in this paper.

\section{References:}

[1]. Freeman, K. C. ,(1970), Ap. J.,160, 811

[2]. Rubin, V. C., Ford, W. K., (1970), Ap, J. 150 , 379

[3]. Combes, F., Boisse',P., Mazure ,A. and Blanchard, A., Galaxies and Cosmology,(2001)

[4]. Ostriker, J.P. ,Peebles, P.J.E., and Yahil,A.,(1974), Ap.J.193, L1

[5]. Einasto, J., Kaasik,A. and Saar,E., (1974),Nature, 250, 309

[6]. Rubin, V.C.,(1993),Proc. Nat. Acad. Sci., 90, 4814

[7]. Sanders, R., H., (2002) Annu. Rev. Astron. Astrophys. Vol 40,p.263-317

[8]. Schwarzschild, K., (1916),Sitz. Acad. Wiss., Physik-Math., Kl. 1, 189

[9]. Einstein, A., (1916), Ann. Physik, 49, 769

[10]. Sofue, Y., Rubin, V.C., Annu.Rev.Astronom.Astrophys.2001,Vol 39, p. 137-174

[11]. Lang, K.R.,(1998), Astrophysical Formula, Vol. 2

[12]. Tinsley, B., (1981), Mon. Not. Roy. Astron. Soc., 194, 63

[13]. Eddington, A.S. (1923), Mathematical Theory of Relativity.

Figures

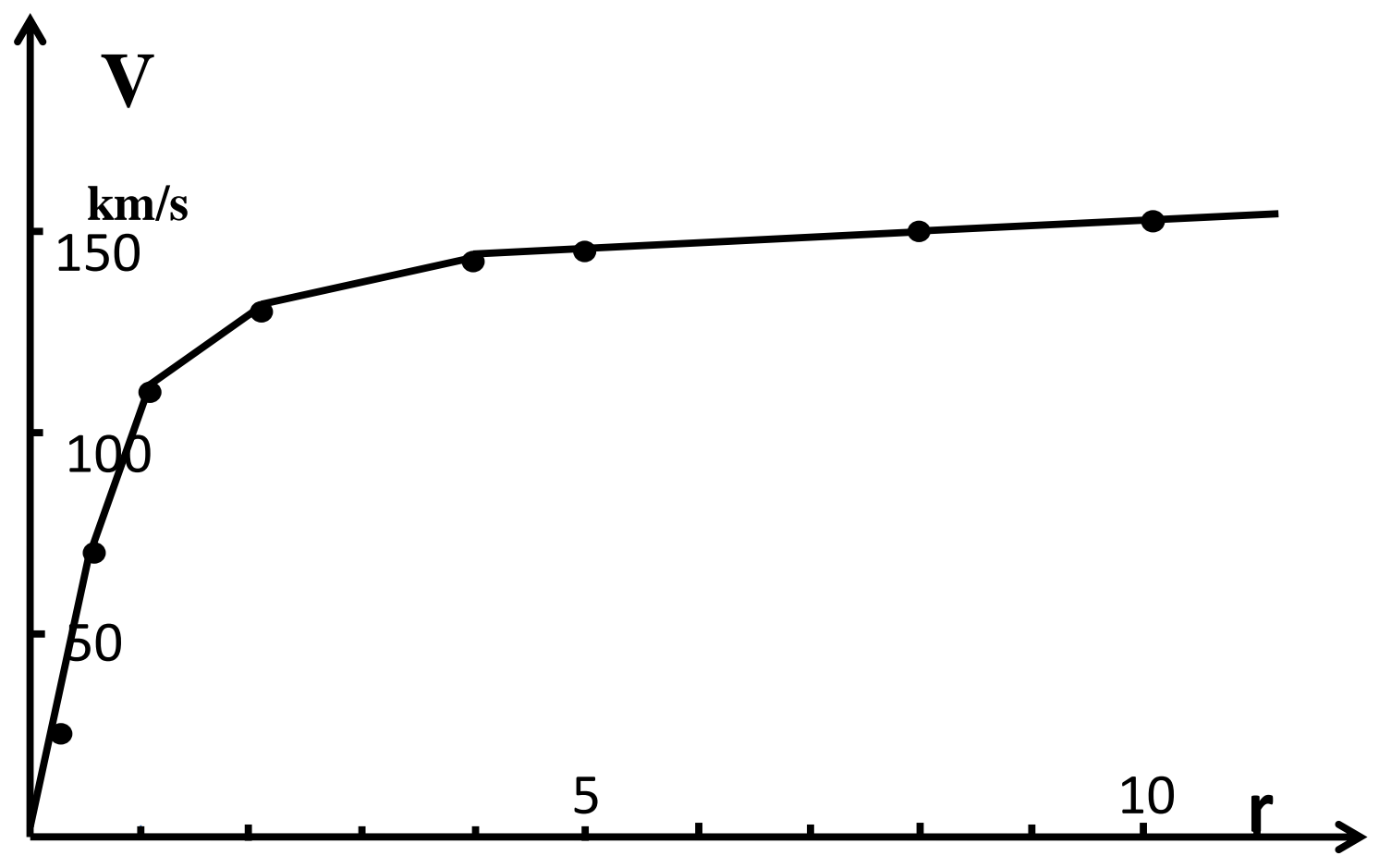

Fig 1

Theoretical graph of rotational speed versus distance in the galaxy according to equation (19) 


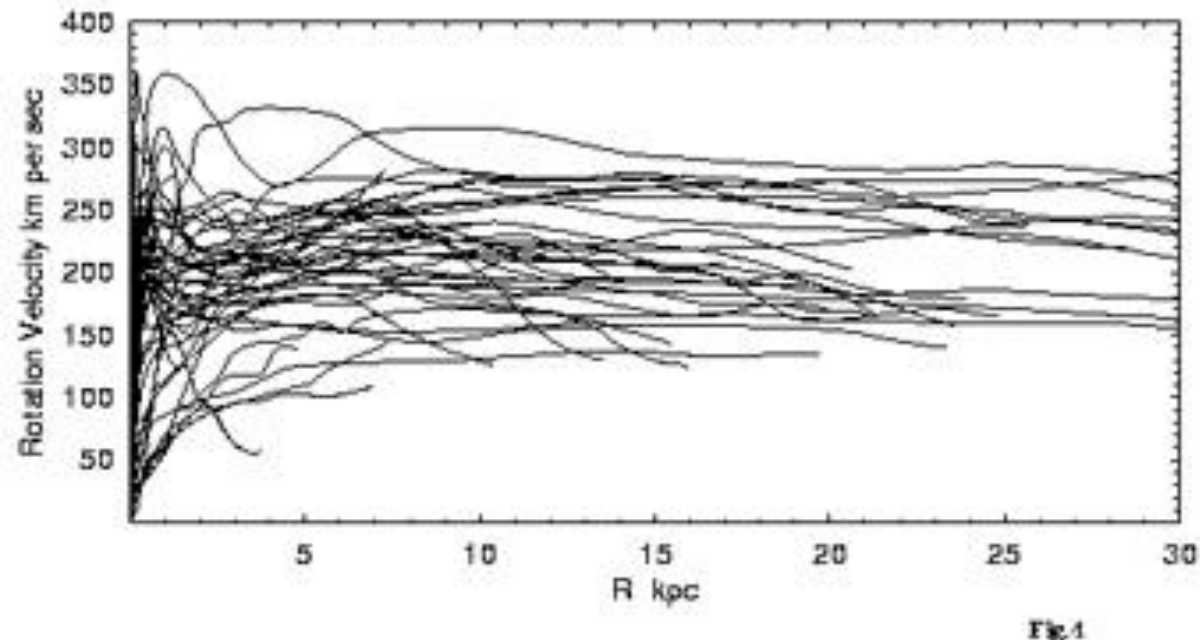

Fig 2

Rotation curves of spiral galaxies obtained by combining $\mathrm{CO}$ data for the central regions, optical for disks, and HI for outer disk and halo Sofue et al.(10)).

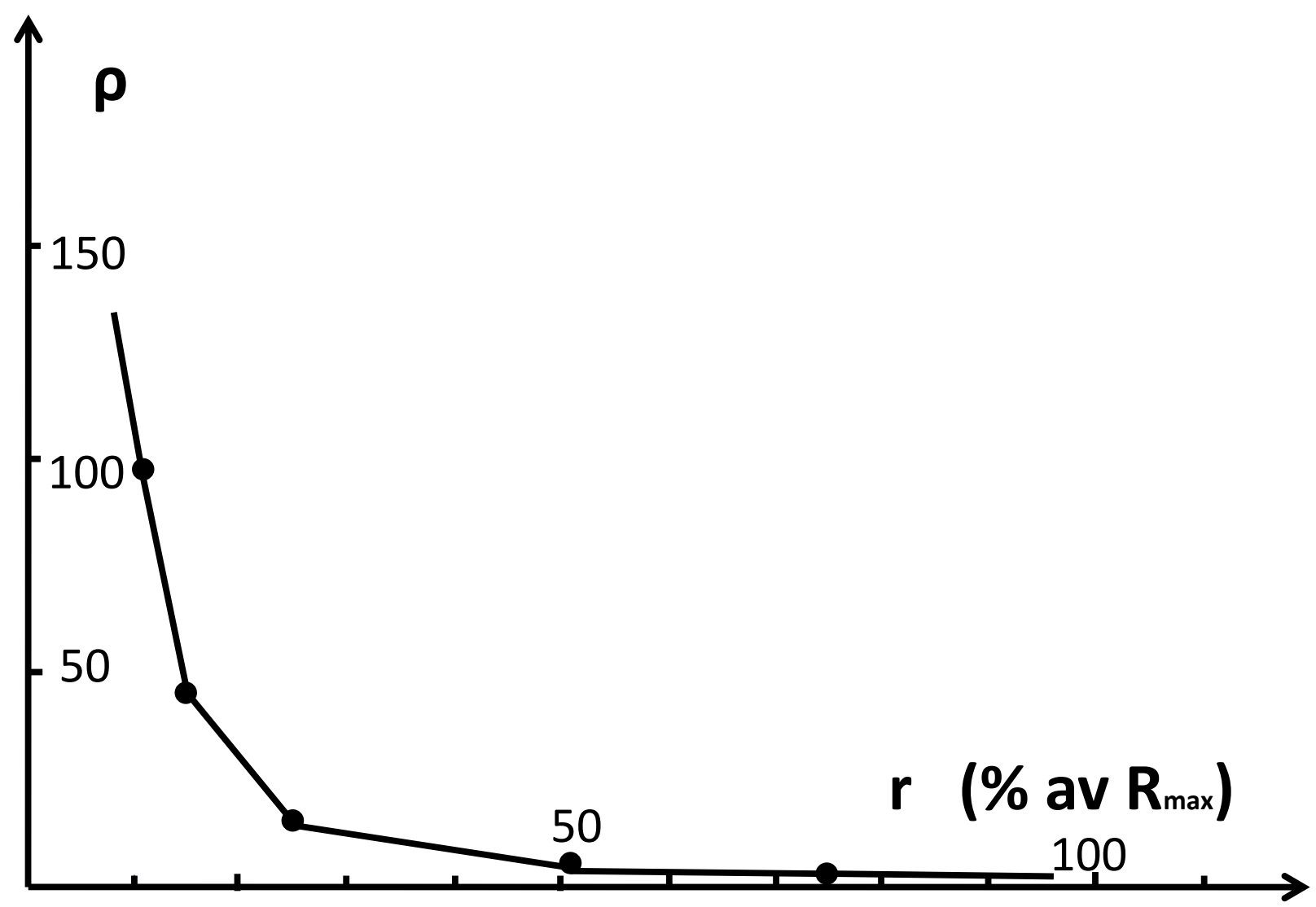

Fig 3

Theoretical graph of density versus distance in the galaxy according to equation (22). The distance is here the ratio between distance $r$ and maximum distance $\mathbf{R}_{\max }$ in percent. 


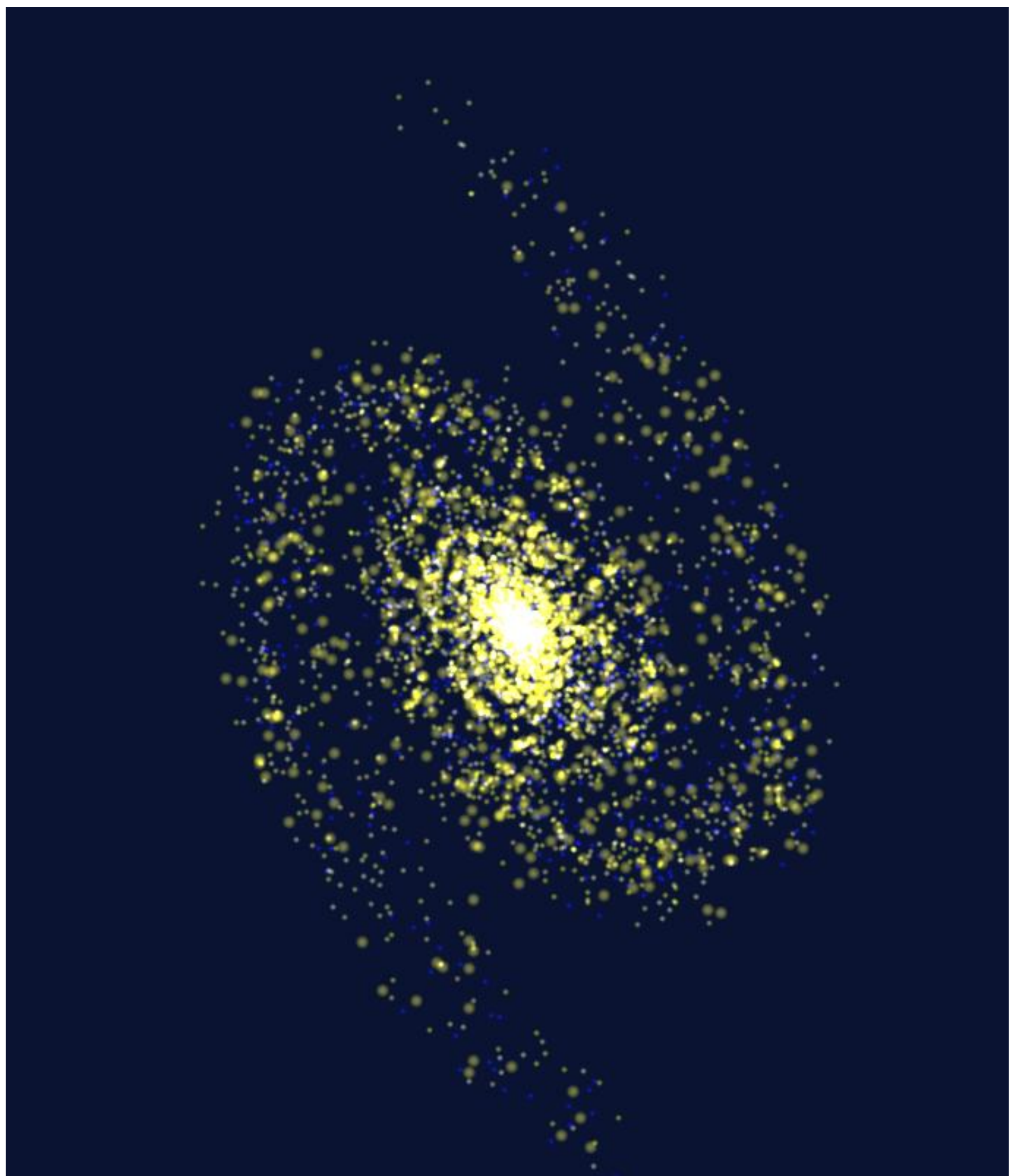

Fig 4

Computer simulations of randomly distributed 6000 "stars" distributed like an ellipsoid. After a while a spiral structure is appeared according to observation and equation (19). 


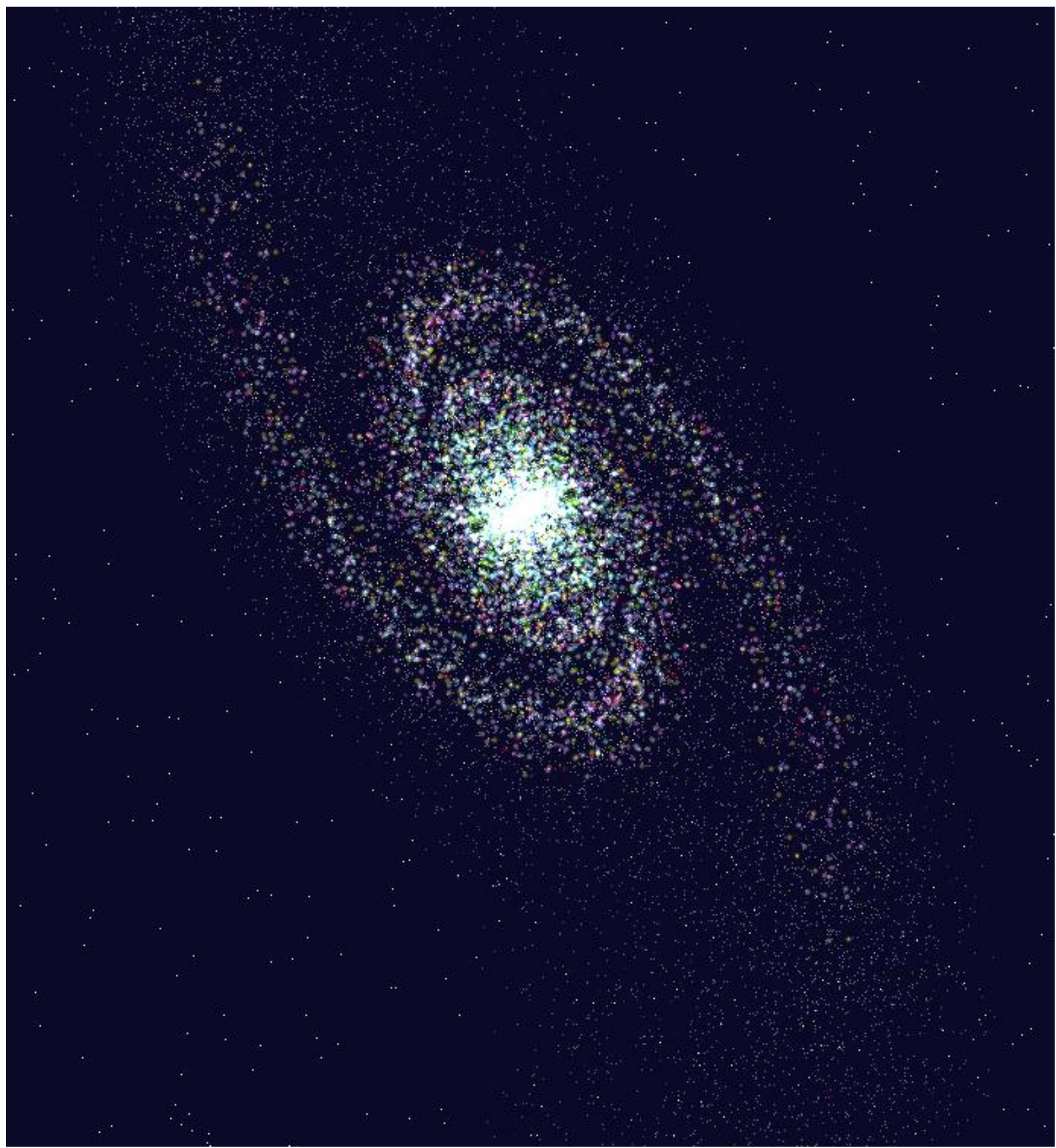

Fig 5

Computer simulations of randomly distributed 30000 "stars" distributed like an ellipsoid. After a while a spiral structure is appeared according to observation and equation (19). 


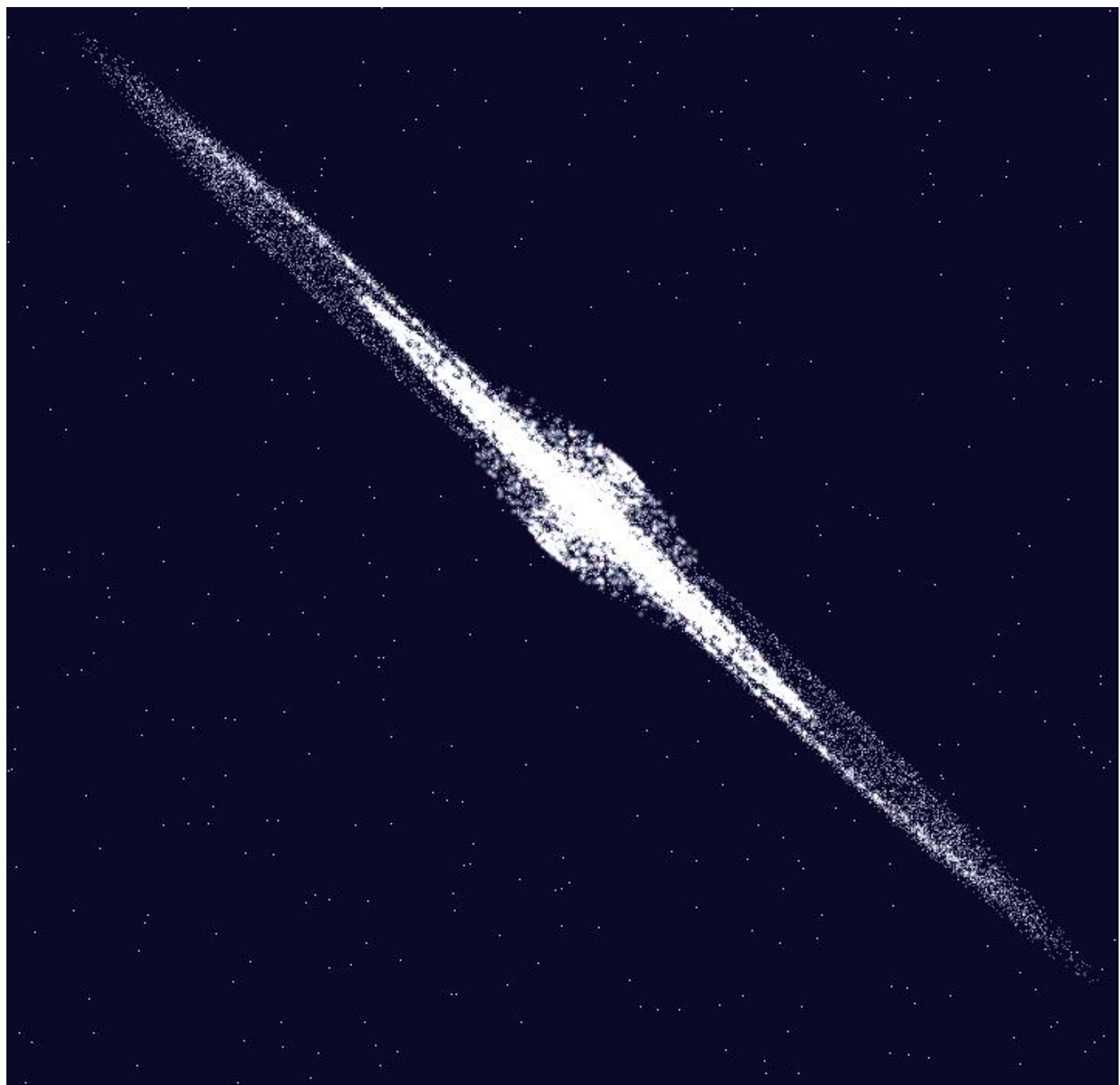

Fig 6

Here the same distribution as Fig 5 but from the side. A halo is also appeared after a while. This is what is observed in most galaxies from the side. 